\title{
The right ventricle
}

\section{P Motshabi Chakane iD}

Department of Anaesthesia, University of the Witwatersrand, South Africa

Corresponding author, email: palesa.motshabi@wits.ac.za

Keywords: right ventricle, ventricular interdependence, coronary perfusion

\section{Distinguishing anatomic features of the right ventricle}

The healthy right ventricle (RV) has a thin-walled structure compared to the thick-walled left ventricle (LV). It has a complex shape that appears crescentic when viewed in cross section and triangular when viewed from the side (Figure 1). ${ }^{1}$

The $\mathrm{RV}$ is made of two layers of muscle that form a 3-dimensional (3D) network of fibres, unlike the LV which is made up of three layers (obliquely muscle superficially, longitudinal in the subendocardium, and predominantly circular in between). ${ }^{2}$ Superficial RV muscle fibres are arranged circumferentially (Figure 1), and the deep fibres are arranged longitudinally. ${ }^{1,2}$ The $\mathrm{RV}$ wall is mainly composed of superficial and deep muscle layers. The superficial muscle layer is circumferential in continuity with the LV, functionally binding the two ventricles. The deep muscles are longitudinal and align base to apex. ${ }^{1}$

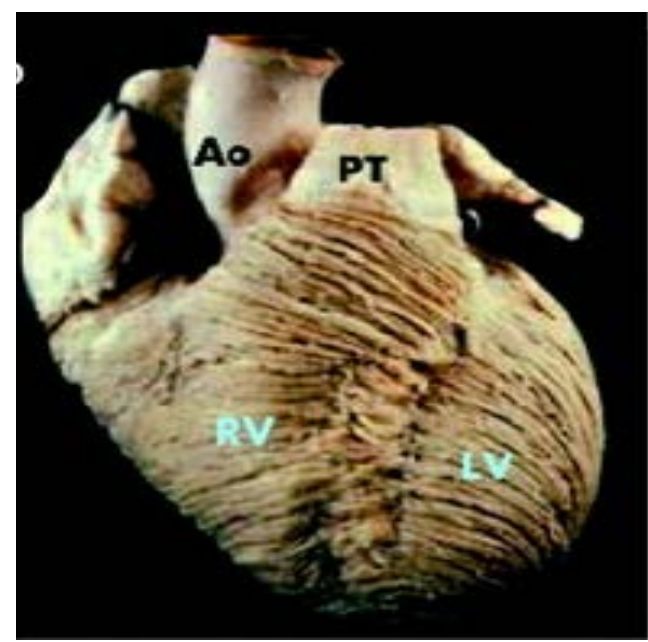

Figure 1: Superficial RV muscle fibres arranged circumferentially ${ }^{1,2}$

The distinction between the two ventricles allows for identity in congenital anomalies. In these conditions there is commonly discordance between the ventricles and the major vessels connected to them. Normally, the RV is located on the right side of the heart and connects with the pulmonary circulation, the LV to the left and posteriorly and connected to the aorta. The

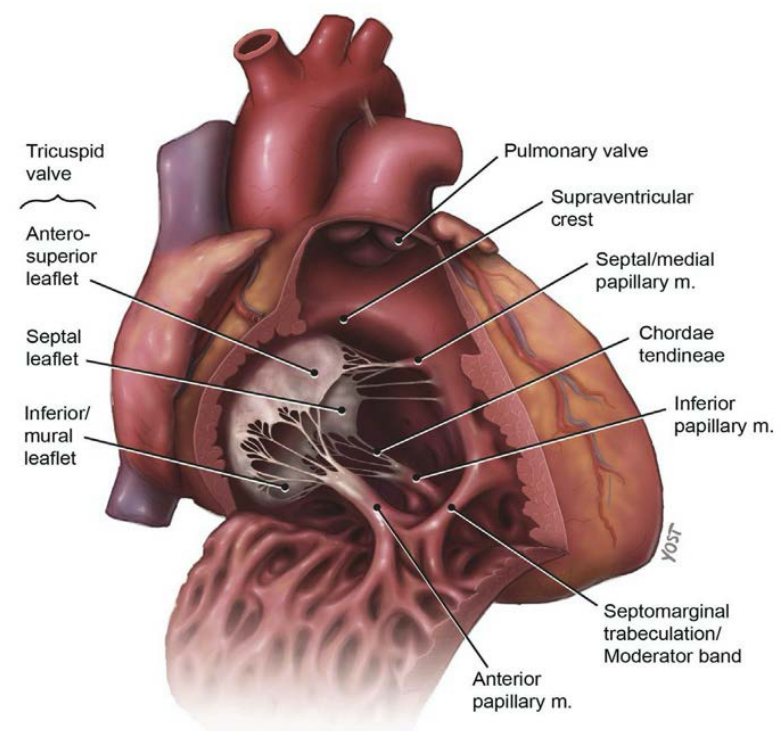

Figure 2: Internal structures of the RV showing the anatomic plane with the moderator band ${ }^{3}$

$\mathrm{RV}$ is a hollow muscular structure with extensive trabeculation. It extends from the inferior border of the right atrium to the cardiac apex, horizontally to make up the inferior border of the heart.

The interventricular septum makes up the left and posterior wall of the RV. Its inflow tract consists of the right atrium and the tricuspid valve whilst outflow is through the infundibulum to the pulmonary trunk. These parts were proposed by Goor and Lillehi in $1977 .^{3}$

The RV has a distinguishing moderator band (Figure 2). There are more than three papillary muscles and a trileaflet configuration of the tricuspid valve with distinguishing septal papillary attachments on the right. It weighs $25 \pm 5 \mathrm{~g} / \mathrm{m}^{2}$ and is approximately $2-5 \mathrm{~mm}$ in thickness, which is $1 / 3$ to $1 / 6$ the thickness of the LV.

\section{Right ventricular physiology}

\section{Myocardial supply and demand}

The right coronary artery (RCA) supplies blood to the right ventricle, the right atrium, and the sinoatrial (SA) and atrioventricular (AV) nodes (Figure 3). The RCA divides into smaller 


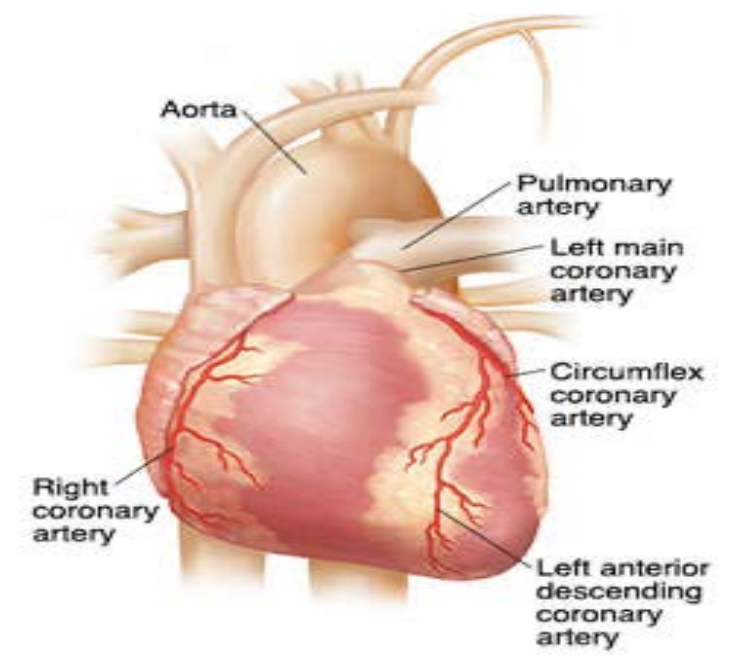

Figure 3: Anatomy of coronary perfusion

branches, including the right posterior descending artery (PDA) and the acute marginal artery. Together with the left anterior descending artery, the PDA helps supply blood to the middle or septum of the heart. RV coronary perfusion occurs throughout the cardiac cycle. ${ }^{3}$

Left coronary artery flow is predominantly in diastole only. Under steady-state conditions, the Fick equation can be used to calculate myocardial oxygen uptake $\left(\mathrm{MVO}_{2}\right)$, such that:

$\mathrm{MVO}_{2}=\mathrm{MBF} \times\left(\mathrm{CaO}_{2}-\mathrm{CvO}_{2}\right)$

where MBF is myocardial blood flow and $\left(\mathrm{CaO}_{2}-\mathrm{CvO}_{2}\right)$ is the coronary arteriovenous oxygen content difference.

Oxygen extraction in the LV is nearly maximal (70-80\%) under baseline conditions; thus, an increase in LV oxygen uptake is essentially dependent on a proportional increase in blood flow. ${ }^{3}$ Myocardial oxygen extraction $\left(\mathrm{MEO}_{2}\right)$, as a percentage, is calculated from measurements of $\mathrm{CaO}_{2}$ and $\mathrm{CvO}_{2}$ :

\section{$\mathrm{MEO}_{2}=\left[\left(\mathrm{CaO}_{2}-\mathrm{CvO}_{2}\right) / \mathrm{CaO}_{2}\right] \times 100$}

In contrast, right coronary blood flow occurs during systole and diastole (Figure 4). The RV perfusion has reduced myocardial oxygen uptake, blood flow, and oxygen extraction. Therefore, there is some oxygen extraction reserve that can be recruited to at least partially offset a reduction in coronary blood flow. The RV has the ability to downregulate its metabolic demand during coronary hypoperfusion and thereby maintain contractile function and energy stores in a transmurally uniform fashion. ${ }^{4}$

The right coronary circulation has a relatively poor autoregulatory capability. Thus, a reduction in right coronary perfusion pressure, which occurs as a consequence of an epicardial coronary stenosis, is accompanied by a nearly proportional decrease in coronary blood flow. However, because of the relatively thin RV wall, a concomitant decrease in wall stiffness occurs, resulting in a reduction in internal work and oxygen demand and an increased oxygen utilisation efficiency, thus preserving oxygen supply-demand balance. ${ }^{4}$

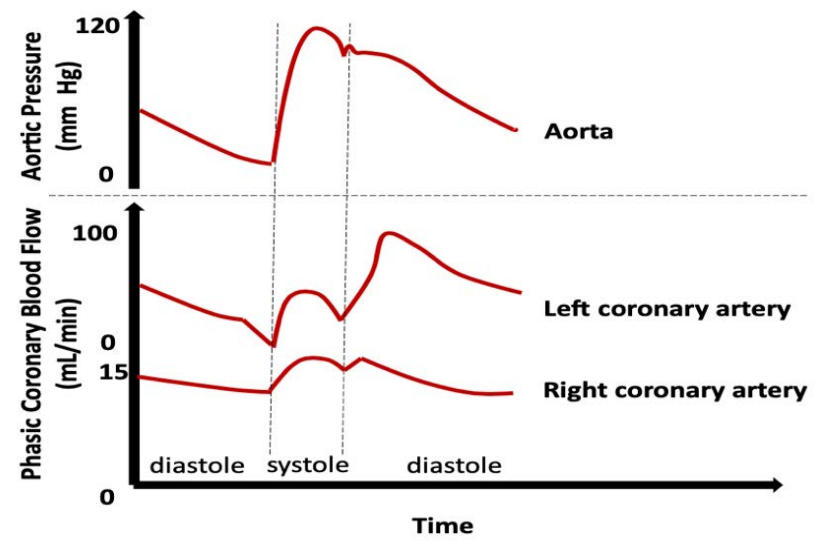

Figure 4: Relationship between coronary blood flow and aortic pressure $^{5}$

The RV enjoys extensive collateral connections from the left coronary circulation and possible retrograde perfusion from the right ventricular cavity through the Thebesian veins. These mechanisms are important in providing protection to the RV against ischaemia-induced dysfunction and injury, but they are less functional in the face of increased may be acute or chronic afterload. Acute increases in pulmonary arterial pressure reduces right ventricular stroke volume. The risk of RV ischaemia is heightened during chronic elevations in RV afterload because microvascular growth fails to match myocyte hypertrophy, and microvascular dysfunction may also be present.

The right coronary circulation is more sensitive than the left to a-adrenergic-mediated constriction, which may contribute to its greater propensity for coronary vasospasm. This characteristic of the right coronary circulation may increase its vulnerability to coronary vasoconstriction and impaired right ventricular perfusion during administration of a-adrenergic receptor agonists. This effect may be manifested during arterial baroreceptor reflex-mediated activation of the sympathetic system secondary to arterial hypotension, or during exercise. Under conditions of a diminished RV vasodilator reserve (e.g. RV hypertrophy or coronary atherosclerotic disease, or when the RV inotropic reserve is compromised), the administration of an adrenergic agonist, such as norepinephrine, for circulatory support may result in coronary vasoconstriction, a reduction in coronary blood flow, and an initiation or exacerbation of myocardial ischaemia in the RV. ${ }^{1,4,6}$

In an in vitro study by Currigan et al., ${ }^{7}$ it was found that vasopressor drugs, commonly used to treat systemic hypotension and maintain organ perfusion, may also induce regional vasoconstriction in specialised vascular beds such as pulmonary vessels. An increase in pulmonary vascular tone may adversely affect patients with pre-existing increased afterload and pump failure. In the study, contractile responses of norepinephrine, phenylephrine, metaraminol and vasopressin were tested on isolated pulmonary and radial artery ring segments, mounted in organ baths. Sympathomimetic-based vasopressor agents constricted both human radial and pulmonary arteries with similar potency, whereas vasopressin, a potent vasoconstrictor 

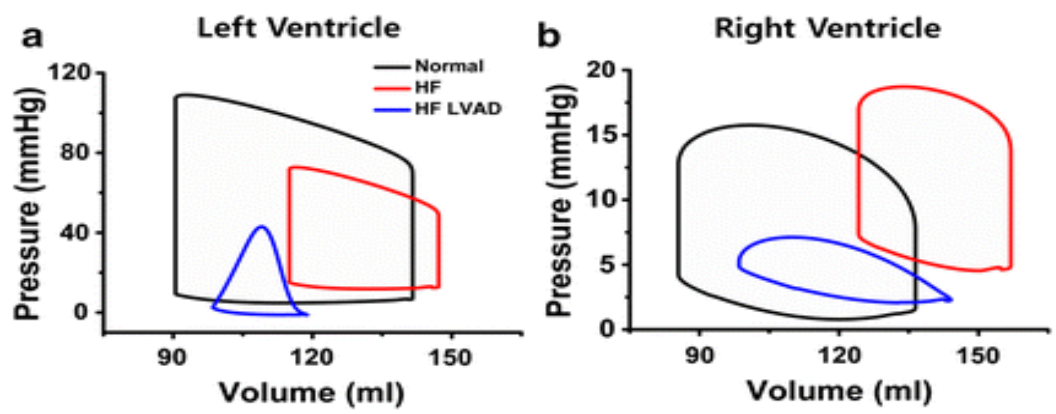

Figure 5: RV pressure-volume (PV) relationships. Normal LV and RV PV loops showing differences in the magnitude of pressure and contrasting the isovolumic phases of LV contraction compared with non-isovolumic contraction in the RV ${ }^{5}$

of radial vessels, had no effect on pulmonary vascular tone. These findings are important particularly in the treatment of hypotension in an ischaemic failing RV challenged by increased

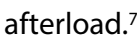

\section{Right ventricular pressure volume loops}

$\mathrm{RV}$ isovolumetric contraction time is shorter than that of the LV as during early systole, the pulmonary valve opens when RV pressure exceeds that of the low-pressure PA. The point of endsystole is less well defined in the RV, leading the RV pressure volume loop to appear almost triangular in shape. This occurs as ejection of blood from the RV can continue despite decreasing RV pressure because of the momentum of blood in the low-pressure system; as a result, the isovolumetric ventricular relaxation phase is shortened or absent in the RV (Figure 5). ${ }^{8}$

The pressure volume loop of a pressure overloaded RV demonstrates post-systolic shortening, and highest increases in endsystolic elastance (Ees), end-diastolic elastance (Eed), and arterial elastance (Ea) (Figure 6).

The cardiomyopathic RV (arrhythmogenic cardiomyopathy in the figure) demonstrates decreased Ees. The pressure overloaded ventricle generates a higher end-systolic pressure (Pes) for a similar stroke volume.

Stroke volume and cardiac output from both the $\mathrm{RV}$ and the LV chambers is equal. Because the $\mathrm{RV}$ is coupled with the normally low-pressure and compliant pulmonary arterial tree, it has significantly less stroke work compared to the LV. Under normal physiologic conditions, the RV handles increased volume easily by increasing RV stroke volume. It has a slightly greater enddiastolic volume, but slightly lower ejection fraction. Regulation of RV contractility in the healthy RV is similar to the LV and is dependent on factors such as heart rate, the Frank-Starling mechanism (stroke volume increases as preload increases), and autonomic neural input.

\section{Ventricular interdependence}

Ventricular interdependence describes the phenomenon whereby the function, volume or pressure in one ventricle influences the function the other ventricle. The intimate anatomic relationship of the RV and LV, with continuity of interlacing muscle bundles including their shared structures, such as the interatrial septum, interventricular septum, and shared coronary blood flow, is the basis for ventricular interdependence. This continuous interplay between the two ventricles represents the anatomic basis of free ventricular wall traction caused by LV contraction. This arrangement contributes to the more complex movement of the LV, which includes torsion, translation, rotation, and thickening. ${ }^{6,10}$

The pericardium encasing the two ventricles also contributes to ventricular interdependence. It does not expand significantly in response to sudden stresses. The two ventricles within the pericardium function like two pumps in series, one against the

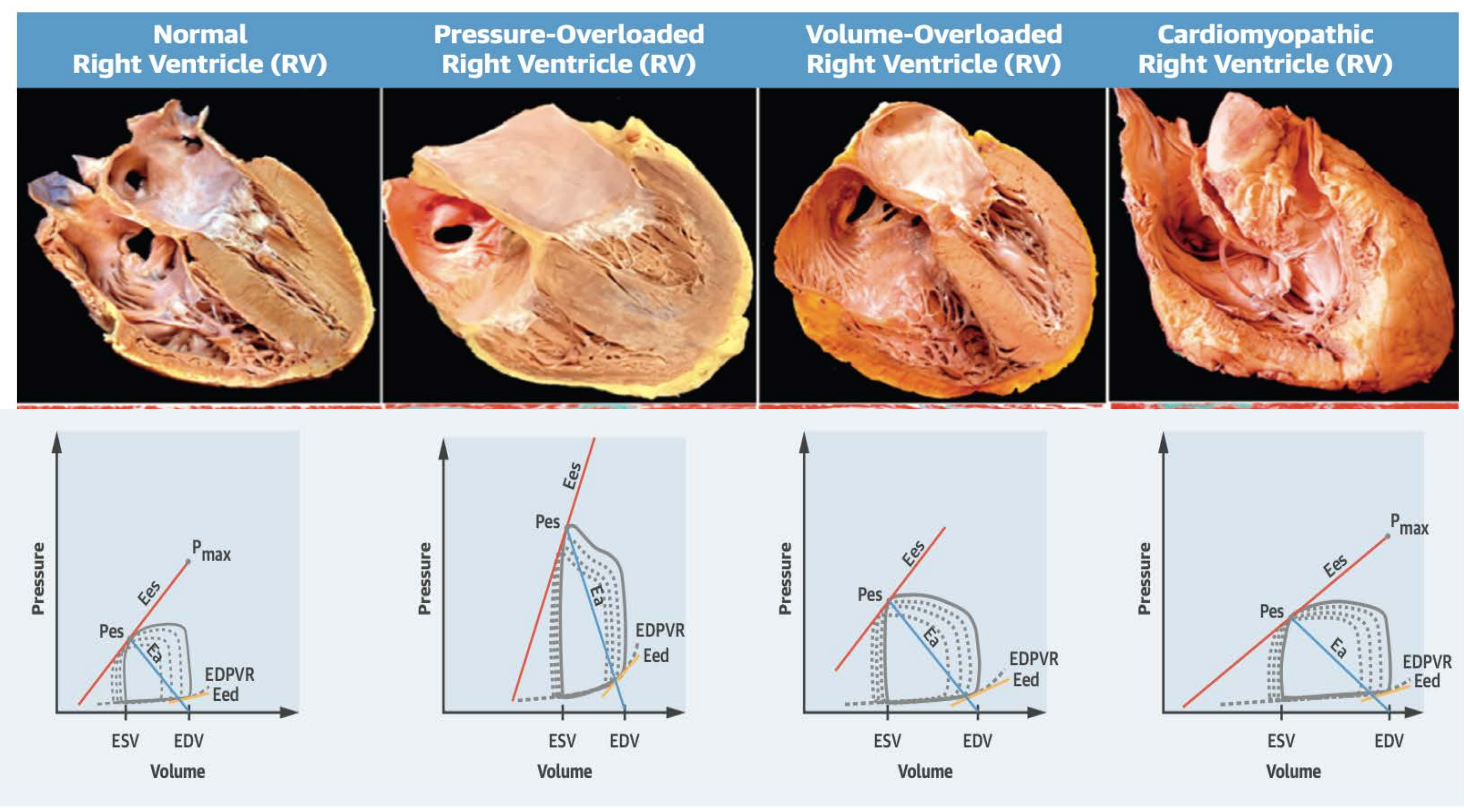

Figure 6: RV pressure volume loops normal and in disease (EDPVR - end-diastolic pressure-volume relationship, EDV - end-diastolic volume, ESV end-systolic volume, LV - left ventricle, Pes - end-systolic pressure, PVC - pulmonic valve closure, RV - right ventricular) ${ }^{9}$ 
A

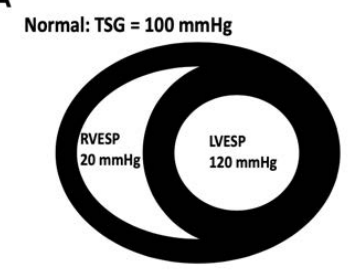

C Elevated PA pressures: TSG $=20 \mathrm{mmHg}$

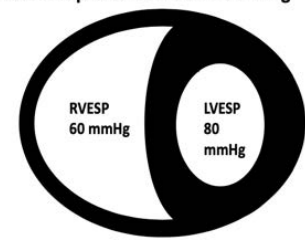

B

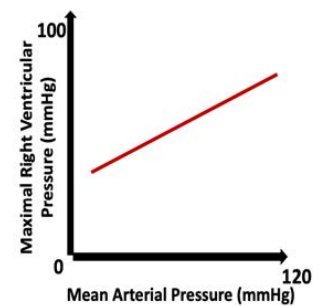

D LVAD: TSG $=-30 \mathrm{mmHg}$

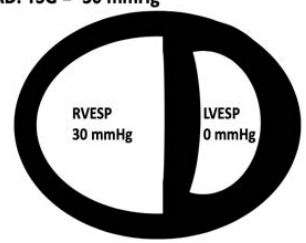

Figure 7: Relationship between transseptal gradient (TSG) and right ventricle $(R V)$ function ${ }^{5}$

highly compliant low pressure pulmonary vasculature and the other against less compliant systemic circulation. ${ }^{6}$

Normal intrapericardial pressure is negative or approximately equal to zero because pericardial pressure varies with pleural pressure at the same hydrostatic level. The pericardium mediates a diastolic interaction whereby one chamber can influence the filling of the opposite chamber. Interestingly, this effect is not completely abolished in the absence of the pericardium. ${ }^{6}$ Hypertrophy, dilatation and alterations in compliance of one ventricle could compress the other ventricle, resulting in diminished function and output. 111,12

The transseptal pressure gradient (TSG) is an important factor in ventricular interdependence and informs the function of the interventricular septum and its effect on the function of the two ventricles (Figure 7). The normal TSG is $100 \mathrm{mmHg}$ (normal range: $80-120 \mathrm{mmHg}$ ). At this pressure gradient, the shape and position of interventricular septum is maintained ( $A$ in Figure 7). RV pressure development is dependent on LV pressure. With elevated RV afterload RVESP $=60 \mathrm{mmHg}$, LVESP is reduced and TSG $=20 \mathrm{mmHg}$, the septum is flattened toward the LV and the $\mathrm{RV}$ is dilated. ${ }^{5}$

When RV pressure or volume overload occurs, the RV can affect LV performance and result in a decreased LV preload and contractility. In normal hearts, LVEDP usually exceeds RVEDP. In times of RV pressure overload, RVEDP may exceed LVEDP forcing the ventricular septum towards the LV during diastole. This distorts the normal LV shape creating a so called 'D-shaped' ventricular cavity (Figure 7), reducing LV diastolic compliance and impairing LV filling. To optimise the TSG in RV pressure overload, PVR must be reduced and LV pressure must be increased. Vasopressin is the ideal vasopressor agent because it increases BP and has minimal effects on pulmonary vascular resistance $(\mathrm{PVR}){ }^{5}$

\section{The right ventricle in disease}

Systemic hypotension in combination with RV ischaemia and high afterload may result in the "lethal combination" of RV

dilatation, interventricular septal bulging, insufficient left ventricular filling, reduced stroke volume, and further systemic hypotension. ${ }^{1}$ However, the acute increase in pulmonary afterload may distend the right heart chambers at the expense of the left-sided chambers and cause pericardial restraint, if total heart volume increases beyond the compliant phase of the pericardial pressure-volume curve. ${ }^{12-17}$

RV dilation and wall thinning result in increased RV wall stress that, along with increased heart rate, leading to further increases in myocardial oxygen consumption, decreased myocardial perfusion, and RV ischaemia. As the RV dilates, severe tricuspid regurgitation may develop, further compromising RV cardiac output and LV filling. RV dilation in the setting of an intact pericardium compromises LV filling by shifting the interventricular septum toward the LV and reducing LV filling and cardiac output. ${ }^{12-17}$

Table I shows normal right sided parameters. Possible causes of increased pulmonary arterial pressure (PAP) can be inferred from the following formula:

\section{Mean PAP $=\mathbf{L A P}+\mathbf{C O} \times \mathbf{P V R}$}

where LAP - left atrial pressure, CO - cardiac output $=(S V \times H R)$, PVR - pulmonary vascular resistance.

Table I: Right sided normal values ${ }^{5}$

\begin{tabular}{ll}
\hline Left atrial pressure & $8 \mathrm{~mm} / \mathrm{Hg}$ \\
\hline Systolic pulmonary artery pressure & $25 \mathrm{~mm} / \mathrm{Hg}$ \\
\hline Diastolic pulmonary artery pressure & $10 \mathrm{~mm} / \mathrm{Hg}$ \\
Mean pulmonary artery pressure & $15 \mathrm{~mm} / \mathrm{Hg}$ \\
\hline Transpulmonary gradient & $7 \mathrm{~mm} / \mathrm{Hg}$ \\
\hline Pulmonary vascular resistance & $0.9-1.4$ wood units or \\
& $90-120$ dynes $\times \mathrm{s} \times \mathrm{cm}^{5}$ \\
\hline
\end{tabular}

When there has been a chronic increase in PVR, RV hypertrophy occurs. The increased thickness reduces wall stress through Laplace's law:

Wall stress $=($ pressure $\mathrm{x}$ internal radius $) /(2 \mathrm{x}$ wall thickness $)$

RV hypertrophy and dilatation produce a significant increase in RV myocardial oxygen consumption. In contrast to the LV, the thinner-walled RV is subjected to greater wall tension for the same degree of increase in end-diastolic volume, leading to increased RV myocardial oxygen demand. Under normal circumstances, the RV intramyocardial pressure is lower than the aortic root pressure. During periods of elevated RV intramyocardial pressure, more coronary flow occurs during diastole, making the RV vulnerable to systemic hypotension, which worsens the mismatch between oxygen demand and supply, and can precipitate ischaemia even in the absence of coronary occlusive disease. Taken together, these factors result in an imbalance between RV myocardial oxygen demand and supply. ${ }^{12-17}$ 


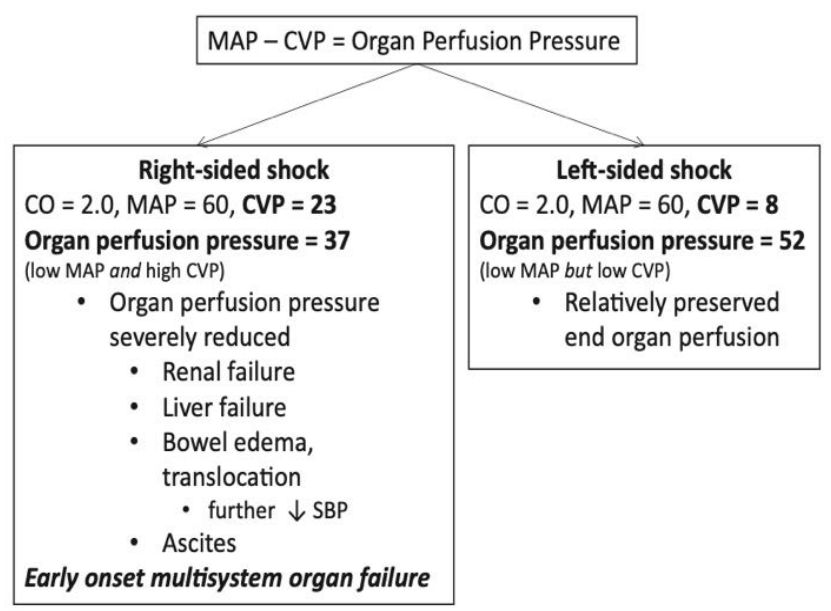

Figure 8: Representation of organ perfusion in LV and RV failure ${ }^{5}$

Tissue perfusion is affected severely in RV failure compared to LV failure. Organ perfusion pressure is determined from the equation:

\section{Organ perfusion pressure $=\mathrm{MAP}-\mathrm{CVP}$}

where MAP - mean arterial pressure and CVP - central venous pressure.

During RV failure, organ perfusion pressure is reduced due to elevated CVP leading to early multi-organ failure, compared to relative preservation of organ function in LV failure (Figure 8).

\section{Conflict of interest}

The author declares no conflict of interest.

\section{Funding source}

None.

\section{ORCID}

P Motshabi Chakane (iD https://orcid.org/0000-0001-9990-6336

\section{References}

1. Haddad F, Hunt SA, Rosenthal DN, Murphy DJ. Right ventricular function in cardiovascular disease, part I: anatomy, physiology, aging, and functional assessment of the right ventricle. Circulation. 2008;117:1436-48. https://doi. org/10.1161/CIRCULATIONAHA.107.653576.

2. Ho SY, Nihoyannopoulos P. Anatomy, echocardiography, and normal right ventricular dimensions. Heart. 2006;92 Suppl 1:i2-13. https://doi.org/10.1136/ hrt.2005.077875.

3. Wang JMH, Rai R, Carrascoc M, et al. An anatomical review of the right ventricle. Translational Research in Anatomy. 2019;17:100049. https://doi.org/10.1016/j. tria.2019.100049.

4. Crystal GJ, Pagel PS. Right ventricular perfusion: physiology and clinical implications. Anesthesiology. 2018;128:202-18. https://doi.org/10.1097/ ALN.0000000000001891.

5. Hrymak C, Strumpher J, Jacobsohn E. Acute right ventricle failure in the intensive care unit: assessment and management. Can J Cardiol. 2017;33:61-71. https:// doi.org/10.1016/j.cjca.2016.10.030.

6. Dell'Italia LJ. Anatomy and physiology of the right ventricle. Cardiol Clin. 2012;30:167-87. https://doi.org/10.1016/j.ccl.2012.03.009.

7. Currigan DA, Hughes RJ, Wright CE, Angus AA, Soeding PF. Vasoconstrictor responses to vasopressor agents in human pulmonary and radial arteries: an in vitro study. Anesthesiology. 2014;121:930-6. https://doi.org/10.1097/ ALN.0000000000000430.

8. Kapur NK, Bader YH. Percutaneous circulatory assist devices for right ventricular failure. Interv Cardiol Clin. 2013;2:445-56. https://doi.org/10.1016/j. iccl.2013.04.001.

9. Sanz J, Sanchez-Quintana D, Bossone E, Bogaard HJ, Naeije R. Anatomy, function and dysfunction of the right ventricle: JACC State-of-the-Art Review. J Am Coll Cardiol. 2019;73:1463-82. https://doi.org/10.1016/j.jacc.2018.12.076.

10. Dell'Italia LJ. The right ventricle: anatomy, physiology, and clinica importance. Curr Prob Cardiol. 1991;16:653-720. https://doi. org/10.1016/0146-2806(91)90009-Y.

11. Walker LA, Buttrick PM. The right ventricle: biologic insights and response to disease: updated. Curr Cardiol Rev. 2013;9:73-81. https://doi.org/10.2174/15734 03X11309010009.

12. Rich S. Right ventricular adaptation and maladaptation in chronic pulmonary arterial hypertension. Cardiol Clin. 2012;30:257-69. https://doi.org/10.1016/j. ccl.2012.03.004.

13. Burghuber OC, Bergmann H. Right-ventricular contractility in chronic obstructive pulmonary disease: a combined radionuclide and hemodynamic study. Respiration. 1988;53:1-12. https://doi.org/10.1159/000195389.

14. Hilde JM, Skjorten I, Grotta OJ, et al. Right ventricular dysfunction and remodeling in chronic obstructive pulmonary disease without pulmonary hypertension. J Am Coll Cardiol. 2013;62:1103-11. https://doi.org/10.1016/j. jacc.2013.04.091.

15. Rubin LJ. Cor pulmonale revisited. From Ferrer and Harvey to the present. Ann Am Thorac Soc. 2018;15(Suppl 1):S42-S4. https://doi.org/10.1513/ AnnalsATS.201710-772KV.

16. Matthay RA, Berger HJ, Davies RA, Loke J. Right and left ventricular exercise performance in chronic obstructive pulmonary disease: radionuclide assessment. Ann Intern Med. 1980;93:234-9. https://doi. org/10.7326/0003-4819-93-2-234.

17. Rapaport E, Wong M, Escobar EE, Martinez G. The effect of upright posture on right ventricular volumes in patients with and without heart failure. Am Heart J. 1966;71:146-52. https://doi.org/10.1016/0002-8703(66)90177-3. 